\title{
On Two Equivalent Dilation Theorems in VH-Spaces
}

\author{
Aurelian Gheondea • Baris Evren Ugurcan
}

Received: 7 December 2010 / Accepted: 24 August 2011 / Published online: 16 September 2011

(C) Springer Basel AG 2011

\begin{abstract}
We prove that a generalized version, essentially obtained by R.M. Loynes, of the B. Sz.-Nagy's Dilation Theorem for $\mathcal{B}^{*}(\mathcal{H})$-valued (here $\mathcal{H}$ is a VH-space in the sense of Loynes) positive semidefinite maps on $*$-semigroups is equivalent with a generalized version of the W.F. Stinespring's Dilation Theorem for $\mathcal{B}^{*}(\mathcal{H})$-valued completely positive linear maps on $B^{*}$-algebras. This equivalence result is a generalization of a theorem of F.H. Szafraniec, originally proved for the case of operator valued maps (that is, when $\mathcal{H}$ is a Hilbert space).
\end{abstract}

\section{Introduction}

In 1955, two of the most celebrated noncommutative dilation theorems have been obtained: one of them refers to operator valued positive semidefinite maps on

Dedicated to F.H. Szafraniec on the occasion of his 70th anniversary.

Communicated by Guest Editors L. Littlejohn and J. Stochel.

A. Gheondea acknowledges support of CNCSIS_Program Idei.

B. E. Ugurcan was supported by TÜBİTAK-BİDEB 2210-Yurtiçi Yüksek Lisans Burs Programı.

A. Gheondea $(\varangle) \cdot$ B. E. Ugurcan

Department of Mathematics, Bilkent University, Bilkent, 06800 Ankara, Turkey

e-mail: aurelian@fen.bilkent.edu.tr

A. Gheondea

Institutul de Matematică al Academiei Române, C.P. 1-764, 014700 Bucharest, Romania

e-mail: A.Gheondea@imar.ro

Present Address:

B. E. Ugurcan

Department of Mathematics, Cornell University, 310 Malott Hall, Ithaca, NY 14853-4201, USA

e-mail: beu4@cornell.edu; ugurcan@math.cornell.edu 
*-semigroups and was proven by Sz.-Nagy in [27], while the other one refers to operator valued completely positive maps on $C^{*}$-algebras and was proven by Stinespring in [24]. Both of these theorems have been motivated by Naĭmark's dilation theorems $[18,19]$ and are constructions of GNS type. However, these theorems have been considered as covering nonlinear and, respectively, linear dilations and hence, pointing out in different directions. Moreover, there is one more important difference between these two theorems: in the Sz.-Nagy's Dilation Theorem, apart of the quite natural positive semidefiniteness condition which appears in the Stinespring's Dilation Theorem as well, there is an additional boundedness condition that shows up and which is essential, unless the $*$-semigroup is actually a group.

Sz.-Nagy's Dilation Theorem can be used for many purposes in operator theory and noncommutative harmonic analysis but, apart from triggering the powerful theory of functional models in nonselfadjoint operator theory of Sz.-Nagy and Foiaş [28], it remained somehow in the shadow for quite a long time. On the contrary, Stinespring's Dilation Theorem made a very successful career and we can see it showing up in most of the works on operator algebras whenever complete positivity plays an important role. As a noncommutative generalization of Naĭmark's dilation theorems, it actually made a more successful career in connection to operator systems, see e.g. Paulsen [21] and Effros and Ruan [7], and quantum operations, that are special types of completely positive maps, e.g. see Davies [5] and Hayashi [9].

It is relatively easy to see that Sz.-Nagy's Dilation Theorem implies Stinespring's Dilation Theorem, due to the fact that the extra boundedness condition in the Sz.-Nagy's theorem is automatically satisfied for completely positive maps on $C^{*}$-algebras. What is not that easy to see is that, actually, these two theorems are equivalent: this is the contents of a theorem of Szafraniec in [26]. In showing this, there are three main steps to take: the first step is to show that the constants in the Sz-Nagy's boundedness condition can be organized in a submultiplicative sequence $c$ with some additional properties (this step was first done in [25]), the next step is to linearize a positive semidefinite map on the $*$-semigroup $\Gamma$ to a positive semidefinite map on a weighted $\ell_{c}^{1}(\Gamma)$ algebra that can be naturally organized as a $B^{*}$-algebra, while the last step is to extend this linear positive semidefinite map to the enveloping $C^{*}$-algebra. Due to an idea of W.B. Arveson, e.g. see his recent survey article [2], the third step can be short-cut by proving the Stinespring's Dilation Theorem for operator valued maps on $B^{*}$-algebras.

On the other hand, there are some generalizations of Hilbert spaces to the case when the inner product takes vector values. Historically, the first one refers to Hilbert modules over $C^{*}$-algebras, initiated by Kaplansky in [11] and showing a tremendous impetus during the last thirty years given by the works of Paschke [20] and Rieffel [23], cf. the monographs $[13,17]$. A generalization to Hilbert $C^{*}$-module operator valued maps of the Stinespring's Theorem has been obtained by Kasparov [12]. Following the same chain of ideas and motivated by questions in operator algebras and mathematical physics one also considered Hilbert modules over locally $C^{*}$-algebras, cf. Inoue [10] and Phillips [22]. A different generalization, motivated by questions in multivariable stochastic processes, was performed by Loynes, see [14-16]. These vector valued Hilbert spaces, that have been acronymed by $\mathrm{VH}$-spaces, show many common features with Hilbert spaces but there are many anomalies as well, the most 
notable ones due to missing a Schwarz Inequality and an analog of the Riesz's Representation Theorem. It is worth noting that $\mathrm{VH}$-spaces are so general that contain Hilbert modules over either $C^{*}$ or locally $C^{*}$-algebras. We refer to $[3,8,29,30]$ for applications of this theory.

In this article we first prove a slightly more general variant of the generalized version of the Sz.-Nagy Dilation Theorem due to Loynes [14] by dropping the assumption that the map is unital, cf. Theorem 3.3. We present a detailed proof of the enhanced Sz.-Nagy-Loynes Theorem due to at least three reasons: one reason is that we need many constructions made during that proof, the second reason is that the obstructions due to the anomalies in operator theory on $\mathrm{VH}$-spaces have to be treated carefully, and the third reason is that the proof employs beautiful ideas from harmonic analysis that are worth to emphasize.

We then prove (twice) a generalized version of Stinespring's Dilation Theorem, first as a consequence of Sz.-Nagy-Loynes Theorem and the second time following the ideas in the original proof in [24], cf. Theorem 4.4. The main result of this paper is Theorem 4.5 when we prove that these $\mathcal{B}^{*}(\mathcal{H})$-valued generalizations are equivalent, and we thus extend Szafraniec's Theorem to this setting. A preliminary section on $\mathrm{VH}$-spaces and their operator theory is included in order to recall the basic notions and facts from this theory. Some of the recalled results have proofs, for the reader's convenience.

We express our thanks to the anonymous referee for the very careful reading of our manuscript and the remarks that improved the presentation.

\section{Notation and Preliminary Results}

In this section we review most of the definitions and some theorems on VH-spaces and their operator theory, cf. Loynes, [14-16].

\subsection{VH-Spaces.}

A complex vector space $Z$ is called admissible if:

(a1) $Z$ is a complete locally convex space.

(a2) $Z$ has an involution $*$, that is, a map $Z \ni z \mapsto z^{*} \in Z$ that is conjugate linear $\left((\alpha x+\beta y)^{*}=\bar{\alpha} x^{*}+\bar{\beta} y^{*}\right.$ for all $\alpha, \beta \in \mathbb{C}$ and all $\left.x, y \in Z\right)$ and involutive $\left(\left(z^{*}\right)^{*}=z\right.$ for all $\left.z \in Z\right)$.

(a3) In $Z$ there is a convex cone $Z_{+}\left(\alpha x+\beta y \in Z_{+}\right.$for all numbers $\alpha, \beta \geq 0$ and all $\left.x, y \in Z_{+}\right)$, that is closed, strict $\left(Z_{+} \cap-Z_{+}=\{0\}\right)$, and consisting of selfadjoint elements only $\left(z^{*}=z\right.$ for all $\left.z \in Z_{+}\right)$. This cone is used to define a partial order in $Z$ by: $z_{1} \geq z_{2}$ if $z_{1}-z_{2} \in Z_{+}$.

(a4) The topology of $Z$ is compatible with the partial ordering in the sense that there exists a base of the topology, linearly generated by a family of neighbourhoods $\left\{N_{j}\right\}_{j \in \mathcal{J}}$ of the origin, such that all of them are convex and solid, that is, whenever $x \in N_{j}$ and $0 \leq y \leq x$ then $y \in N_{j}$.

It can be proven that axiom (a4) is equivalent with the following one: 
$\left(\mathrm{a} 4^{\prime}\right)$ There exists a collection of seminorms $\left\{p_{j}\right\}_{j \in \mathcal{J}}$ defining the topology of $Z$ that are increasing, that is, $0 \leq x \leq y$ implies $p_{j}(x) \leq p_{j}(y)$.

If, in addition, to the axioms (a1)-(a4), the space $Z$ satisfies also the following:

(a5) With respect to the specified partial ordering, any bounded monotone sequence is convergent.

then $Z$ is called a strongly admissible space.

In order to substantiate this definition we recall a few relevant examples.

Example 2.1 (a) $C^{*}$-Algebras. If $\mathcal{A}$ is a $C^{*}$-algebra then it is an admissible space with the cone $\mathcal{A}^{+}=\left\{a^{*} a \mid a \in \mathcal{A}\right\}$ of positive elements and the underlying normed topology. In particular, this is the case for the $C^{*}$-algebra $\mathcal{B}(H)$ of all bounded linear operators on a complex Hilbert space $H$, as well as for the $C^{*}$-algebra $C(K)$ of all complex valued continuous functions on a compact Hausdorff space $K$. See, e.g., Dixmier [6].

(b) Locally $C^{*}$-Algebras. A complex $*$-algebra $\mathcal{A}$ is a locally $C^{*}$-algebra if it is endowed with a family of seminorms $\left\{p_{\alpha}\right\}_{\alpha \in A}$ that are submultiplicative, that is, $p_{\alpha}(x y) \leq p_{\alpha}(x) p_{\alpha}(y)$ for all $x, y \in \mathcal{A}$ and all $\alpha \in A$, they satisfy the $C^{*}$-algebra condition, that is, $p_{\alpha}\left(x^{*} x\right)=p_{\alpha}(x)^{2}$ for all $x \in \mathcal{A}$ and all $\alpha$, and $\mathcal{A}$ is complete with respect to the topology induced by this family of seminorms. The notion of positive element is the same as in the case of a $C^{*}$-algebra. Clearly, any locally $C^{*}$-algebra is an admissible space. See Inoue [10].

(c) $\mathcal{B}\left(X, X^{\prime}\right)$. Let $X$ be a complex Banach space and $X^{\prime}$ its topological conjugate dual. On the vector space $\mathcal{B}\left(X, X^{\prime}\right)$ of all bounded linear operators $T: X \rightarrow X^{\prime}$, a natural notion of positive operator can be defined: $T$ is positive if $(T x)(x) \geq 0$ for all $x \in X$. Let $\mathcal{B}_{+}\left(X, X^{\prime}\right)$ be the collection of all positive operators and note that it is a strict cone that is closed with respect to the weak operator topology. The involution $*$ in $\mathcal{B}\left(X, X^{\prime}\right)$ is defined in the following way: for any $T \in \mathcal{B}\left(X, X^{\prime}\right)$, $T^{*}=T^{\prime} \mid X$, that is, the restriction to $X$ of the conjugate dual operator $T^{\prime}: X^{\prime \prime} \rightarrow$ $X^{\prime}$. With respect to the weak operator topology, the cone $\mathcal{B}_{+}\left(X, X^{\prime}\right)$, and the involution $*$ just defined, $\mathcal{B}\left(X, X^{\prime}\right)$ becomes an admissible space. See Weron [29], as well as D. Gaşpar and P. Gaşpar [8].

Given a complex linear space $\mathcal{E}$ and an admissible space $Z$, a $Z$-valued inner product or $Z$-gramian is, by definition, a mapping $\mathcal{E} \times \mathcal{E} \ni(x, y) \mapsto[x, y] \in Z$ subject to the following properties:

(ve1) $[x, x] \geq 0$ for all $x \in \mathcal{E}$, and $[x, x]=0$ if and only if $x=0$.

(ve2) $[x, y]=[y, x]^{*}$ for all $x, y \in \mathcal{E}$.

(ve3) $\left[a x_{1}+b x_{2}, y\right]=a\left[x_{1}, y\right]+b\left[x_{2}, y\right]$ for all $a, b \in \mathbb{C}$ and all $x_{1}, x_{2} \in \mathcal{E}$.

A complex linear space $\mathcal{E}$ onto which a $Z$-valued inner product $[\cdot, \cdot \cdot]$ is specified, for a certain admissible space $Z$, is called a $V E$-space (Vector Euclidean space).

In any VE-space $\mathcal{E}$ over an admissible space $Z$ the familiar polarization formula

$$
4[x, y]=\sum_{k=0}^{3} \mathrm{i}^{k}\left[x+\mathrm{i}^{k} y, x+\mathrm{i}^{k} y\right], \quad x, y \in \mathcal{E},
$$


holds, which shows that the $Z$-valued inner product is perfectly defined by the $Z$-valued quadratic map $\mathcal{E} \ni x \mapsto[x, x] \in Z$.

Any VE-space $\mathcal{E}$ can be made in a natural way into a Hausdorff separated locally convex space by considering the weakest locally convex topology on $\mathcal{E}$ that makes the mapping $E \ni h \mapsto[h, h] \in Z$ continuous, more precisely, letting $\left\{N_{j}\right\}_{j \in \mathcal{J}}$ be the collection of convex and solid neighbourhoods of the origin in $Z$ as in axiom (a4), the collection of sets

$$
U_{j}=\left\{x \in \mathcal{E} \mid[x, x] \in N_{j}\right\}, \quad j \in \mathcal{J},
$$

is a topological base of neighbourhoods of the origin of $\mathcal{E}$ that linearly generates the weakest locally convex topology on $\mathcal{E}$ that makes the mapping $\mathcal{E} \ni h \mapsto[h, h] \in Z$ continuous, cf. Theorem 1 in [14]. In terms of seminorms, this topology can be defined in the following way: let $\left\{p_{j}\right\}_{j \in \mathcal{J}}$ be a family of increasing seminorms defining the topology of $Z$ and let

$$
q_{j}(h)=p_{j}([h, h])^{1 / 2}, \quad h \in \mathcal{E}, j \in \mathcal{J} .
$$

Then the specified topology of $\mathcal{E}$ is fully determined by the family of seminorms $\left\{q_{j}\right\}_{j \in \mathcal{J}}$.

If $\mathcal{E}$ is complete with respect to this locally convex topology then it is called a $V H$-space (Vector Hilbert space). In case the admissible space $Z$ is strongly admissible, a VH-space is called an LVH-space (Limit Vector Hilbert space), cf. [15], or Loynes space, cf. $[3,30]$. LVH-spaces are more suitable for spectral representations of their unitary or selfadjoint operators, but we do not use them in this article.

The concept of $V E$-spaces isomorphism is also naturally defined: this is just a linear bijection $U: \mathcal{E} \rightarrow \mathcal{F}$, for two VE-spaces over the same admissible space $Z$, such that, $[U x, U y]=[x, y]$ for all $x, y \in \mathcal{E}$. Any VE-space $\mathcal{E}$ can be embedded as a dense subspace of a $\mathrm{VH}$-space $\mathcal{H}$, uniquely determined up to an isomorphism, cf. Theorem 2 in [14].

In order to substantiate these definitions we recall a few examples.

Example 2.2 $C^{*}$-Algebras. Let $\mathcal{A}$ be a $C^{*}$-algebra. As in Example 2.1, $\mathcal{A}$ is an admissible space. Then note that letting $[x, y]=y^{*} x$ we have an $\mathcal{A}$-gramian on $\mathcal{A}$ with respect to which $\mathcal{A}$ becomes a $\mathrm{VH}$-space.

Hilbert $C^{*}$-Modules. Let $\mathcal{A}$ be a $C^{*}$-algebra. An inner product $\mathcal{A}$-module is a linear space $\mathcal{E}$ which is a right $\mathcal{A}$-module together with a map $\mathcal{E} \times \mathcal{E} \ni(x, y) \mapsto\langle x, y\rangle \in \mathcal{A}$ such that: (i) $\langle x, y a+z b\rangle=\langle x, y\rangle a+\langle x, z\rangle b$, (ii) $\langle x, y a\rangle=\langle x, y\rangle a$, (iii) $\langle y, x\rangle=$ $\langle x, y\rangle^{*}$, (iv) $\langle x, x\rangle \geq 0$ and if $\langle x, x\rangle=0$ then $x=0$. A norm on $\mathcal{E}$ can be given by $\|x\|=\|\langle x, x\rangle\|$ and, if $\mathcal{E}$ is complete with respect to this norm then $\mathcal{E}$ is called a Hilbert $C^{*}$-module. Clearly, any Hilbert $C^{*}$-module is a $\mathrm{VH}$-space (there is a difference with respect to our definition since, traditionally, Hilbert $C^{*}$-modules are linear in the second variable, but this can be remedied). These objects are intensively studied, e.g. see the monographs $[13,17]$.

Hilbert Modules over Locally $C^{*}$-Algebras. In the above definition, one can replace the $C^{*}$-algebra $\mathcal{A}$ by a locally $C^{*}$-algebra and get the notion of Hilbert modules over locally $C^{*}$-algebras. Again, this is an example of a $\mathrm{VH}$-space. 
In general VH-spaces, an analog of the Schwarz Inequality does not hold. However, some of its consequences can be proven using slightly different techniques. One such consequence is the following lemma.

Lemma 2.3 (Loynes [14]) Let $Z$ be an admissible space, $\mathcal{E}$ a complex vector space and $[\cdot, \cdot]: \mathcal{E} \times \mathcal{E} \rightarrow Z$ a positive semidefinite sesquilinear map, that $i s,[\cdot, \cdot]$ is linear in the first variable and conjugate linear in the second variable, and $[x, x] \geq 0$ for all $x \in \mathcal{E}$. If $f \in \mathcal{E}$ is such that $[f, f]=0$, then $\left[f, f^{\prime}\right]=\left[f^{\prime}, f\right]=0$ for all $f^{\prime} \in \mathcal{E}$.

Proof Fix $f \in \mathcal{E}$ such that $[f, f]=0$ and let $\lambda \in \mathbb{C} \backslash\{0\}$ and $f^{\prime} \in \mathcal{E} \backslash\{0\}$ be arbitrary. We have

$$
\left[f+\lambda f^{\prime}, f+\lambda f^{\prime}\right]=[f, f]+\lambda\left[f^{\prime}, f\right]+\bar{\lambda}\left[f, f^{\prime}\right]+|\lambda|^{2}\left[f^{\prime}, f^{\prime}\right]
$$

hence, since $[f, f]=0$ it follows

$$
=\lambda\left[f^{\prime}, f\right]+\bar{\lambda}\left[f, f^{\prime}\right]+|\lambda|^{2}\left[f^{\prime}, f^{\prime}\right] \geq 0 .
$$

We claim that

$$
\lambda\left[f^{\prime}, f\right]+\bar{\lambda}\left[f, f^{\prime}\right]=0 .
$$

Indeed, if this is not true then note that we can replace $f$ by $t f$ for any real $t$ and get

$$
t\left(\lambda\left[f^{\prime}, f\right]+\bar{\lambda}\left[f, f^{\prime}\right]\right) \geq-|\lambda|^{2}\left[f^{\prime}, f^{\prime}\right]
$$

and then, letting either $t \rightarrow+\infty$ or $t \rightarrow-\infty$ we immediately get a contradiction with our assumptions that both $\lambda$ and $f^{\prime}$ are not null. Thus, (2.4) is proven.

Now, letting $\lambda$ successively be 1 and $i$ in (2.4) we get

$$
\left[f^{\prime}, f\right]+\left[f, f^{\prime}\right]=0 \text { and } \mathrm{i}\left(\left[f^{\prime}, f\right]-\left[f, f^{\prime}\right]\right)=0 \text {, }
$$

and hence $\left[f^{\prime}, f\right]=\left[f, f^{\prime}\right]=0$.

\subsection{Linear Operators in VH-Spaces}

The collection $\mathcal{L}(\mathcal{E}, \mathcal{F})$ of all linear and continuous operators between VE-spaces $\mathcal{E}$ and $\mathcal{F}$ is naturally organized as a complex vector space. In particular, the set $\mathcal{L}(\mathcal{E})$ of all linear and continuous operators $T: \mathcal{E} \rightarrow \mathcal{E}$ is naturally organized as a complex algebra.

Given two VH-spaces $\mathcal{H}$ and $\mathcal{K}$, a linear operator $A: \mathcal{H} \rightarrow \mathcal{K}$ is called bounded if there exists a constant $k \geq 0$ such that

$$
[A x, A x] \leq k[x, x], \quad x \in \mathcal{H} .
$$


Taking into account the definition of the underlying topology of a $\mathrm{VH}$-space, any linear and bounded operator $T$ is uniformly continuous. In particular, boundedness of a linear operator on a dense linear manifold implies that it can uniquely extended to the whole $\mathrm{VH}$-space, a construction that will be intensively used throughout. We denote the special class of bounded operators by $\mathcal{B}(\mathcal{H}, \mathcal{K})$. For a bounded operator $A \in \mathcal{B}(\mathcal{H}, \mathcal{K})$ we define its operator norm $\|A\|$ by the square root of the least $k$ satisfying (2.5), that is,

$$
\|A\|=\inf \{\sqrt{k} \mid[A x, A x] \leq k[x, x] \text {, for all } x \in \mathcal{H}\}
$$

It is easy to see that the infimum is actually a minimum and hence, that we have

$$
[A x, A x] \leq\|A\|^{2}[x, x], \quad x \in \mathcal{H}
$$

If $\mathcal{H}$ is a $\mathrm{VH}$-space then $\mathcal{B}(\mathcal{H})=\mathcal{B}(\mathcal{H}, \mathcal{H})$ is a Banach algebra with respect to the usual algebraic operations and the operator norm, cf. Theorem 1 in [15].

\subsection{The $C^{*}$-Algebra $\mathcal{B}^{*}(\mathcal{H})$}

Given two VH-spaces $\mathcal{H}$ and $\mathcal{K}$, an operator $A \in \mathcal{B}(\mathcal{H}, \mathcal{K})$ is called adjointable if there exists a bounded operator $A^{*}: \mathcal{K} \rightarrow \mathcal{H}$ such that for all $x \in \mathcal{H}$ and $y \in \mathcal{K}$

$$
[A x, y]=\left[x, A^{*} y\right]
$$

We denote by $\mathcal{B}^{*}(\mathcal{H}, \mathcal{K})$ the collection of all adjointable elements in $\mathcal{B}(\mathcal{H}, \mathcal{K})$. We emphasize the fact that, in a general $\mathrm{VH}$-space setting, not all bounded operators are adjointable. This is mostly due to the lack of an analog of the Riesz Representation Theorem. For example, as noted in [15], letting $\mathcal{K}$ be a compact Hausdorff space, we consider the $C^{*}$-algebra $C(K)$ as a $\mathrm{VH}$-space as in Example 2.2. If $A$ is a linear operator on the VH-space $C(K)$ which possesses an adjoint, then $A$ corresponds to multiplication by some fixed function $\alpha \in C(K)$.

The definitions of selfadjoint, unitary, and normal operators are the same as in the Hilbert space case. It is clear that $A$ is selfadjoint if and only if $[A x, y]=[x, A y]$ for all $x, y \in \mathcal{H}$, and also, by the polarization formula (2.1), this is equivalent to

$$
[A x, x]=[A x, x]^{*}, \quad x \in \mathcal{H} \text {. }
$$

A bounded operator $A$ in $\mathcal{H}$ is called positive if $[A x, x] \geq 0$ for all $x \in \mathcal{H}$. From (2.9) it follows that a positive operator is necessarily selfadjoint. A contraction is a linear operator $T$ such that $[T x, T x] \leq[x, x]$ for all $x \in \mathcal{H}$. By Theorem 2 in [15], the involution $*$ is isometric, that is, $\left\|T^{*}\right\|=\|T\|$. In the following we will need a slightly stronger version of that theorem, as follows. 
Lemma 2.4 Let $T \in \mathcal{B}(\mathcal{H}, \mathcal{K})$ for some $V H$-spaces $\mathcal{H}$ and $\mathcal{K}$ such that there exists a linear operator $S: \mathcal{D} \rightarrow \mathcal{H}$, for some dense subspace $\mathcal{D}$ of $\mathcal{K}$ with the property that

$$
[T h, k]_{\mathcal{K}}=[h, S k]_{\mathcal{H}}, \quad h \in \mathcal{H}, k \in \mathcal{D} .
$$

Then $T$ is adjointable, $T^{*}$ is an extension of $S$, in particular $S$ is bounded as well and $\|T\|=\|S\|$.

Proof We use the fact that, for any $u, v \in \mathcal{K}$ we have

$$
[u-v, u-v]=[u, u]-[u, v]-[v, u]+[v, v] \geq 0
$$

hence

$$
[u, u]+[v, v] \geq[u, v]+[v, u] .
$$

Observe that, without loss of any generality, we can assume that $\|T\| \leq 1$ and then, for any $k \in \mathcal{D}$ we have

$$
\begin{aligned}
{[S k, S k]_{\mathcal{H}} } & =\frac{1}{2}\left([S k, S k]_{\mathcal{H}}+[S k, S k]_{\mathcal{H}}\right) \\
& =\frac{1}{2}\left([k, T S k]_{\mathcal{K}}+[T S k, k]_{\mathcal{K}}\right) \\
& \leq \frac{1}{2}\left([T S k, T S k]_{\mathcal{K}}+[k, k]_{\mathcal{K}}\right) \leq \frac{1}{2}\left([S k, S k]_{\mathcal{H}}+[k, k]_{\mathcal{K}}\right)
\end{aligned}
$$

Observe that the above calculation gives us

$$
[S k, S k]_{\mathcal{H}} \leq[k, k]_{\mathcal{K}}, \text { for all } k \in \mathcal{D}
$$

that is, $S$ is a contraction, hence continuous and it can be extended uniquely to an operator $\tilde{S} \in \mathcal{B}(\mathcal{K}, \mathcal{H})$. Then $(2.10)$ holds for all $k \in \mathcal{K}$ which means that $T$ is adjointable and $T^{*}=\tilde{S}$.

If $A \in \mathcal{B}^{*}(\mathcal{H})$ is selfadjoint, then we have

$$
-\|A\|[x, x] \leq[A x, x] \leq\|A\|[x, x]
$$

The importance of the previous inequality, cf. Theorem 3 in [15], is that, sometimes, it may be used instead of the Schwarz Inequality which, in general, does not hold for a VH-space. Moreover, assume that $A$ is a linear operator in $\mathcal{H}$ and that for some real numbers $m, M$ we have

$$
m[x, x] \leq[A x, x] \leq M[x, x], \quad x \in \mathcal{H} .
$$

Then $A \in \mathcal{B}^{*}(\mathcal{H})$ and $A=A^{*}$. If, in addition, $m$ is the maximum and $M$ is the minimum with these properties, then $\|A\|=\min \{|m|,|M|\}$. 
It is now clear that $\mathcal{B}^{*}(\mathcal{H})$ is a Banach $*$-algebra with isometric involution. According to Theorem 4 in [15], for any VH-space $\mathcal{H}$ the algebra $\mathcal{B}^{*}(\mathcal{H})$ is a $C^{*}$-algebra, precisely, we have $\left\|A^{*} A\right\|=\|A\|^{2}$ for all $A \in \mathcal{B}^{*}(\mathcal{H})$.

On the other hand, the natural cone of positive elements in a $C^{*}$-algebra $\mathcal{A}$ is $\mathcal{A}^{+}=\left\{a^{*} a \mid a \in \mathcal{A}\right\}$. According to Theorem 5 in [15], given $\mathcal{H}$ a VH-space and $A \in \mathcal{B}^{*}(\mathcal{H})$, then $A$ is positive (that is, $[A x, x] \geq 0$ for all $x \in \mathcal{H}$ ) if and only if $A=B^{*} B$ for some $B \in \mathcal{B}^{*}(\mathcal{H})$. So, the two notions coincide.

A subspace $\mathcal{M}$ of a $\mathrm{VH}$-space $\mathcal{H}$ is orthocomplemented or accessible if every element $x \in \mathcal{H}$ can be written as $x=y+z$ where $y$ is in $\mathcal{M}$ and $z$ is such that $[z, m]=0$ for all $m \in \mathcal{M}$, that is, $z$ is in the orthogonal companion $\mathcal{M}^{\perp}$ of $\mathcal{M}$. Observe that if such a decomposition exists it is unique and hence the orthogonal projection $P_{\mathcal{M}}$ onto $\mathcal{M}$ can be defined by $P_{\mathcal{M}} x=y$. Any orthogonal projection $P$ is selfadjoint and idempotent, in particular we have $[P x, y]=[P x, P y]$ for all $x, y \in \mathcal{H}$, hence $P$ is positive and contractive. Conversely, any selfadjoint idempotent operator is an orthogonal projection onto its range subspace. Any orthocomplemented subspace is closed.

\section{Dilations of Positive Semidefinite $\mathcal{B}^{*}(\mathcal{H})$-Valued Maps on $*$-Semigroups}

In this section we prove a generalization of the Sz.-Nagy's Dilation Theorem [27] to VH-spaces, essentially due to Loynes [14]. We will actually prove a slightly more general variant dropping the assumption that the positive semidefinite function is the identity when evaluated at the unit, and for this reason some more technical results are needed.

\section{$3.1 \mathcal{B}^{*}(\mathcal{H})$-Valued Positive Semidefinite Maps on $*$-Semigroups}

Recall that a $*$-semigroup is a (multiplicative) semigroup $\Gamma$ onto which there exists an involution, usually denoted by $*$, that is, $\Gamma \ni \gamma \mapsto \gamma^{*} \in \Gamma$ having the properties: $(\beta \gamma)^{*}=\gamma^{*} \beta^{*}$ and $\left(\gamma^{*}\right)^{*}=\gamma$, for all $\beta, \gamma \in \Gamma$. If $\Gamma$ has a unit $\epsilon$ then $\epsilon^{*}=\epsilon$.

In case $\Gamma$ is a group and we use the multiplicative notation, one can take $\gamma^{*}=\gamma^{-1}$, but other choices are also possible.

Let $\mathcal{H}$ be a VH-space and consider a family $T=\left\{T_{\xi}\right\}_{\xi \in \Gamma}$ of operators in $\mathcal{B}^{*}(\mathcal{H})$ indexed by a $*$-semigroup $\Gamma$. Equivalently, we think $T$ as a function on $\Gamma$ and valued in $\mathcal{B}^{*}(\mathcal{H})$. Given $n$ an arbitrary natural number, we call $T n$-positive if for any $\eta_{1}, \ldots, \eta_{n} \in \Gamma$ and any $h_{1}, \ldots, h_{n} \in \mathcal{H}$, the following inequality holds

$$
\sum_{i, j=1}^{n}\left[T_{\eta_{i}^{*} \eta_{j}} h_{j}, h_{i}\right] \geq 0 .
$$

Clearly, if $T$ is $n$-positive then it is $k$-positive for all natural numbers $k \leq n$. $T$ is called positive semidefinite if it is $n$-positive for all natural numbers $n$. 
Just from the definition it follows that if $T$ is positive semidefinite then $T_{\eta^{*} \eta}$ should be all positive, and hence selfadjoint. However, it is not yet clear why $T$ should be selfadjoint, in the sense that $T_{\xi^{*}}=T_{\xi}^{*}$ for all $\xi \in \Gamma$. To see this we make use of the fact that, just from the definition, we confined VH-spaces to complex spaces.

Proposition 3.1 Let $\mathcal{H}$ be a (complex) VH-space and $T$ a $\mathcal{B}^{*}(\mathcal{H})$-valued function on a unital *-semigroup $\Gamma$. If $T$ is 2-positive then $T$ is selfadjoint, that is, $T_{\xi^{*}}=T_{\xi}^{*}$ for all $\xi \in \Gamma$.

Proof We write the 2-positivity condition (3.1) for $\eta_{1}=\epsilon, \eta_{2}=\eta, h_{1}=x$, and $h_{2}=y$ in order to get

$$
\left[T_{\eta} y, x\right]+\left[T_{\eta^{*}} x, y\right]+\left[T_{\epsilon} x, x\right]+\left[T_{\eta^{*} \eta} y, y\right] \geq 0 .
$$

Since $T$ is 1-positive, the last two terms are positive, hence the sum of the first two terms is in the real span of the cone $Z_{+}(\mathcal{H}$ is a $\mathrm{VH}$-space with admissible space $Z)$, hence it is selfadjoint in $Z$, that is,

$$
\left[T_{\eta} y, x\right]+\left[T_{\eta^{*}} x, y\right]=\left[x, T_{\eta} y\right]+\left[y, T_{\eta^{*}} x\right],
$$

equivalently

$$
\left[\left(T_{\eta^{*}}-T_{\eta}^{*}\right) x, y\right]+\left[y,\left(T_{\eta}^{*}-T_{\eta^{*}}\right) x\right]=0 .
$$

Letting $y=\mathrm{i}\left(T_{\eta^{*}}-T_{\eta}^{*}\right) x$ we obtain

$$
2 \mathrm{i}\left[\left(T_{\eta^{*}}-T_{\eta}^{*}\right) x,\left(T_{\eta^{*}}-T_{\eta}^{*}\right) x\right]=0,
$$

and since $x$ is arbitrary in $\mathcal{H}$, it follows that $T_{\eta^{*}}=T_{\eta}^{*}$.

The operator $T_{\epsilon}$, for a positive semidefinite function on a $*$-semigroup $\Gamma$ with unit $\epsilon$, should be positive. In the original dilation theorems of Sz.-Nagy [27] and its generalization by Loynes [14], it was assumed that $T_{\epsilon}=I$. Dropping this assumption, as simple as it may look, will make some technical obstructions in the VH-space case. For this reason, we first have a look on the implications of taking $T_{\epsilon}=0$. The obstruction we have to overcome is, again, due to a missing Schwarz type inequality.

Lemma 3.2 Let $T$ be a 2-positive function $T: \Gamma \rightarrow \mathcal{B}^{*}(\mathcal{H})$ for some $*$-semigroup $\Gamma$ with unit $\epsilon$ and a VH-space $\mathcal{H}$. If $T_{\epsilon}=0$ then $T_{\eta}=0$ for all $\eta \in \Gamma$.

Proof Since $T$ is 2-positive we have (3.2) and taking into account the assumption $T_{\epsilon}=0$ it follows that

$$
\left[T_{\eta} y, x\right]+\left[T_{\eta^{*}} x, y\right] \geq-\left[T_{\eta^{*} \eta} y, y\right]
$$

Since $T$ is 1 -positive as well, it follows that $\left[T_{\eta^{*} \eta} y, y\right] \geq 0$. We claim that

$$
\left[T_{\eta} y, x\right]+\left[T_{\eta^{*}} x, y\right]=0
$$


Indeed, if $\left[T_{\eta^{*} \eta} y, y\right]=0$ from (3.3) we have $\left[T_{\eta} y, x\right]+\left[T_{\eta^{*}} x, y\right] \geq 0$. Replacing $x$ by $-x$ we get the opposite inequality, hence (3.4) should hold. In case $\left[T_{\eta^{*} \eta} y, y\right]>0$ we note that the rightmost side in (3.3) does not depend on $x$ and hence we can replace $x$ by $t x$, with $t$ arbitrary real number. We proceed further like in the proof of Lemma 2.3 and conclude that $\left[T_{\eta} y, x\right]+\left[T_{\eta^{*}} x, y\right]=0$. Thus, in either cases (3.4) is proven.

To finish the proof, in (3.4) we replace $x$ with i $x$ and get $\left[T_{\eta} y, x\right]-\left[T_{\eta^{*}} x, y\right]=0$, hence from here and (3.4) it follows that $\left[T_{\eta} y, x\right]=0$. Since $x, y \in \mathcal{H}$ are arbitrary this means that $T_{\eta}=0$.

\subsection{A Generalized B. Sz.-Nagy's Dilation Theorem}

We pass now to state and prove the promised generalization of the Sz.-Nagy's Dilation Theorem for $*$-semigroups.

Theorem 3.3 Let $\Gamma$ be a $*$-semigroup with unit $\epsilon$ and $T=\left\{T_{\xi}\right\}_{\xi \in \Gamma} \subseteq \mathcal{B}^{*}(\mathcal{H})$, for some $\mathrm{VH}$-space $\mathcal{H}$. The following assertions are equivalent:

(1) $T$ satisfies the following conditions:

(b) $T$ is positive semidefinite as a function on $\Gamma$, in the sense that for any finitely supported family $\left\{g_{\xi}\right\}_{\xi \in \Gamma}$ in $\mathcal{H}$ we have

$$
\sum_{\xi, \eta \in \Gamma}\left[T_{\xi^{*} \eta} g_{\eta}, g_{\xi}\right] \geq 0
$$

(c) For any $\alpha \in \Gamma$ there exists a nonnegative number $c(\alpha)$ such that for any finitely supported family $g=\left\{g_{\xi}\right\}_{\xi \in \Gamma}$ in $\mathcal{H}$ we have

$$
\sum_{\xi, \eta \in \Gamma}\left[T_{\xi^{*} \alpha^{*} \alpha \eta} g_{\eta}, g_{\xi}\right] \leq c(\alpha)^{2} \sum_{\xi, \eta \in \Gamma}\left[T_{\xi^{*} \eta} g_{\eta}, g_{\xi}\right]
$$

(2) There exists a VH-space $\widehat{\mathcal{H}}$, an operator $V \in \mathcal{B}^{*}(\mathcal{H}, \widehat{\mathcal{H}})$, and a $*$-representation $D=\left\{D_{\xi}\right\}_{\xi \in \Gamma}$ of $\Gamma$ in $\mathcal{B}^{*}(\widehat{\mathcal{H}})$, such that

$$
T_{\xi}=V^{*} D_{\xi} V, \quad \xi \in \Gamma
$$

Moreover, $\widehat{\mathcal{H}}$ can be obtained to be minimal in the sense that it is spanned by elements of the form $D_{\xi} V f$, where $f \in \mathcal{H}$ and $\xi \in \Gamma$ and, in this case, the triple $(\widehat{\mathcal{H}} ; D ; V)$ is uniquely determined up to an isomorphism of VH-spaces that intertwines the $*$-representations and keeps the corresponding operators $V$.

In addition, in case $T_{\epsilon}=I, \mathcal{H}$ can be isometrically embedded as an orthocomplemented subspace into $\widehat{\mathcal{H}}$ and, letting $P_{\mathcal{H}}$ be the orthogonal projection onto $\mathcal{H}$, we have

$$
T_{\xi}=\left.P_{\mathcal{H}} D_{\xi}\right|_{\mathcal{H}}, \quad \xi \in \Gamma .
$$


The proof of this theorem follows closely the lines of the proof of B. Sz.-Nagy for the Hilbert space case but with important differences caused by the anomalies of VH-spaces, when compared to Hilbert spaces. In addition, we point out a harmonic analysis perspective of the constructions in the proof.

Proof $(1) \Rightarrow(2)$ We divide the proof into six steps:

Step 1. Construction of the space $\widehat{\mathcal{H}}$.

We let $\mathcal{G}$ be the space of all finitely supported families $g=\left\{g_{\xi}\right\}_{\xi \in \Gamma}$ of vectors in $\mathcal{H}$. $\mathcal{G}$ has a natural $Z$-valued positive semidefinite sesquilinear pairing $[\cdot, \cdot]_{\mathcal{G}}$

$$
\left[g, g^{\prime}\right]_{\mathcal{G}}=\sum_{\xi \in \Gamma}\left[g_{\xi}, g_{\xi}^{\prime}\right]_{\mathcal{H}}, \quad g, g^{\prime} \in \mathcal{G}
$$

We consider the convolution operator $\mathbf{T}$ on the complex linear space $\mathcal{G}$,

$$
(\mathbf{T} g)_{\xi}=\sum_{\eta \in \Gamma} T_{\xi^{*} \eta} g_{\eta}, \quad g \in \mathcal{G}, \xi \in \Gamma .
$$

Clearly, $\mathbf{T}$ is a linear operator, with codomain the complex vector space of all $\mathcal{H}$-valued maps on $\Gamma$, and let $\mathcal{F}$ denote its range space, that is, the linear space of all families $f=\left\{f_{\xi}\right\}_{\xi \in \Gamma}$ of vectors in $\mathcal{H}$ which have representations

$$
f_{\xi}=(\mathbf{T} g)_{\xi}=\sum_{\eta \in \Gamma} T_{\xi^{*} \eta} g_{\eta}, \quad \text { for some } g \in \mathcal{G} \text { and all } \xi \in \Gamma \text {. }
$$

Note that, actually, the sum in (3.8) also makes sense when at least one of $g$ or $g^{\prime}$ has finite support, the other one can be an arbitrary $\mathcal{H}$-valued map on $\Gamma$. With this remark, since $T$ is positive semidefinite, the convolution operator $\mathbf{T}$ has a symmetry property expressed by

$$
\left[\mathbf{T} g, g^{\prime}\right]_{\mathcal{G}}=\left[g, \mathbf{T} g^{\prime}\right]_{\mathcal{G}}, \quad g, g^{\prime} \in \mathcal{G},
$$

which follows immediately by Proposition 3.1.

We define a $Z$-valued pairing $[\cdot, \cdot]_{\mathbf{T}}$ on $\mathcal{G}$ by

$$
\left[g, g^{\prime}\right]_{\mathbf{T}}=\left[\mathbf{T} g, g^{\prime}\right]_{\mathcal{G}}=\sum_{\xi, \eta \in \Gamma}\left[T_{\xi^{*} \eta} g_{\eta}, g_{\xi}^{\prime}\right]_{\mathcal{H}}, \quad g, g^{\prime} \in \mathcal{G},
$$

and note that, by assumption (b) and Proposition 3.1, this is a positive semidefinite sesquilinear $Z$-valued map but, in general, it is not positive definite. However, by Lemma 2.3 , it is easy to see that

$$
\operatorname{Ker}(\mathbf{T})=\left\{g \in \mathcal{G} \mid[g, g]_{\mathbf{T}}=0\right\}=\left\{g \in \mathcal{G} \mid\left[g, g^{\prime}\right]_{\mathbf{T}}=0 \text { for all } g^{\prime} \in \mathcal{G}\right\},
$$


which shows that, the $Z$-valued pairing $[\cdot, \cdot]_{\mathcal{F}}$ on $\mathcal{F}$, defined by

$$
\left[f, f^{\prime}\right]_{\mathcal{F}}:=\left[\mathbf{T} g, g^{\prime}\right]_{\mathcal{G}}=\sum_{\xi \in \Gamma}\left[f_{\xi}, g_{\xi}^{\prime}\right]_{\mathcal{H}}, \quad f=\mathbf{T} g, f^{\prime}=\mathbf{T} g^{\prime}, g, g^{\prime} \in \mathcal{G},
$$

is correctly defined (that is, independent on the particular representation $f=\mathbf{T} g$ and $\left.f^{\prime}=\mathbf{T} g^{\prime}\right)$ and that, actually, it is a $Z$-valued inner product on $\mathcal{F}$. In order to see positive definiteness, let $f \in \mathcal{F}$ be such that $[f, f]_{\mathcal{F}}=0$. By Lemma 2.3, we get $\left[f, f^{\prime}\right]=0$ for any $f^{\prime} \in \mathcal{F}$. For any $h \in \mathcal{H}$ and any $\eta \in \Gamma$ we let $\delta_{\eta} h \in \mathcal{G}$ be defined by

$$
\left(\delta_{\eta} h\right)_{\xi}= \begin{cases}h, & \xi=\eta \\ 0, & \text { otherwise }\end{cases}
$$

We take $f^{\prime}=\mathbf{T}\left(\delta_{\eta} f_{\eta}\right) \in \mathcal{F}$. By (3.14) and (3.11) we have

$$
\left[f, f^{\prime}\right]_{\mathcal{F}}=\left[f, \delta_{\eta} f_{\eta}\right]_{\mathcal{G}}=\sum_{\xi \in \Gamma}\left[f_{\xi},\left(\delta_{\eta} f_{\eta}\right)_{\xi}\right]_{\mathcal{H}}=\left[f_{\eta}, f_{\eta}\right]_{\mathcal{H}}=0 .
$$

This implies that $f_{\eta}=0$ for any $\eta \in \Gamma$, so $f=0$.

So far, we showed that $\mathcal{F}$ is a VE-space equipped with the vector inner product $[\cdot, \cdot]_{\mathcal{F}}$. By taking the abstract completion of $\left(\mathcal{F},[\cdot, \cdot]_{\mathcal{F}}\right)$ we obtain the VH-space $\widehat{\mathcal{H}}$ as desired.

Step 2. Construction of the operator $V \in \mathcal{B}^{*}(\mathcal{H}, \widehat{\mathcal{H}})$.

We consider the operator $V: \mathcal{H} \rightarrow \widehat{\mathcal{H}}$ defined by

$$
V h=\left\{T_{\xi *} h\right\}_{\xi \in \Gamma} \in \mathcal{F}, \quad h \in \mathcal{H},
$$

more precisely, $(V h)_{\xi}=T_{\xi * h}$ for all $\xi \in \Gamma$. Actually, to see that $V h \in \mathcal{F}$, with the notation as in (3.15), note that

$$
\sum_{\eta \in \Gamma} T_{\xi * \eta}\left(\delta_{\epsilon} h\right)_{\eta}=T_{\xi * h}
$$

that is, $V h=\left\{T_{\xi} * h\right\}_{\xi \in \Gamma}=\mathbf{T}\left(\delta_{\epsilon} h\right) \in \mathcal{F}$.

On the other hand, for any $h \in \mathcal{H}$ we have

$$
\begin{aligned}
{[V h, V h]_{\mathcal{F}} } & =\left[\left\{T_{\xi} h\right\}_{\xi \in \Gamma},\left\{T_{\xi} h\right\}_{\xi \in \Gamma}\right]_{\mathcal{F}} \\
& =\sum_{\xi \in \Gamma}\left[T_{\xi^{*}} h,\left(\delta_{\epsilon} h\right)_{\xi}\right]_{\mathcal{H}} \\
& =\left[T_{\epsilon} h, h\right]_{\mathcal{H}} \leq\left\|T_{\epsilon}\right\|[h, h]_{\mathcal{H}},
\end{aligned}
$$

which proves that $V$ is bounded. Thus, $V$ can be extended by continuity to an operator $V \in \mathcal{B}(\mathcal{H}, \widehat{\mathcal{H}})$. 
Noting that, for arbitrary $f \in \mathcal{F}$ and $h \in \mathcal{H}$ we have

$$
[f, V h]_{\mathcal{F}}=\left[f,\left\{T_{\xi} h\right\}_{\xi \in \Gamma}\right]_{\mathcal{F}}=\sum_{\xi \in \Gamma}\left[f_{\xi},\left(\delta_{\epsilon} h\right)_{\xi}\right]_{\mathcal{H}}=\left[f_{\epsilon}, h\right]_{\mathcal{H}},
$$

we can apply Lemma 2.4 and conclude that $V$ is adjointable. For further reference, let us record that, as a consequence of (3.17), we have

$$
V^{*} f=f_{\epsilon}, \quad f \in \mathcal{F} .
$$

Step 3. Construction of the $*$-representation $D: \Gamma \rightarrow \mathcal{B}^{*}(\widehat{\mathcal{H}})$.

For arbitrary $\xi \in \Gamma, D_{\xi}$ is defined first on the vector space $\mathcal{F}$ : for any $f \in \mathcal{F}$,

$$
D_{\xi} f:=\left\{f_{\xi^{*} \eta}\right\}_{\eta \in \Gamma} \text { that is, }\left(D_{\xi} f\right)_{\eta}=f_{\xi^{*} \eta} \text {, for all } \eta \in \Gamma \text {. }
$$

However, we need to check that the right hand side of (3.19) really belongs to $\mathcal{F}$. More precisely, we need to find a $g^{\xi} \in \mathcal{G}$ such that $D_{\xi} f=\mathbf{T} g^{\xi}$. If we plug the right side of (3.19) into (3.10) we get

$$
f_{\xi^{*} \eta}=\sum_{\gamma \in \Gamma} T_{\eta^{*} \xi \gamma} g_{\gamma}=\sum_{\zeta \in \Gamma} T_{\eta^{*} \zeta} g_{\zeta}^{\xi}
$$

where, for all $\zeta \in \Gamma$,

$$
g_{\zeta}^{\xi}= \begin{cases}0 & \text { if the equation } \xi \gamma=\zeta \text { has no solution } \gamma \in \Gamma \\ \sum_{\xi \gamma=\zeta} g_{\gamma} & \text { otherwise }\end{cases}
$$

hence, since clearly $g^{\xi} \in \mathcal{G}$, from (3.20) it follows that the ranges of all $D_{\xi}$ lie in $\mathcal{F}$.

We first show that $D$ is a representation of $\Gamma$ on $\mathcal{F}$, that is,

$$
D_{\alpha \beta}=D_{\alpha} D_{\beta}, \quad \alpha, \beta \in \Gamma
$$

Let $f \in \mathcal{F}$ and define $g$ by $g_{\eta}=\left(D_{\beta} f\right)_{\eta}=f_{\beta^{*} \eta}$ for all $\eta \in \Gamma$. Then $\left\{D_{\alpha} g\right\}_{\eta \in \Gamma}=$ $\left\{g_{\alpha^{*} \eta}\right\}_{\eta \in \Gamma}$ and $g_{\alpha^{*} \eta}=f_{\beta^{*} \alpha^{*} \eta}=\left(D_{\alpha \beta} f\right)_{\eta}$, hence (3.21) is proven.

We now show that $D$ is actually a $*$-representation on $\mathcal{F}$, that is,

$$
\left[D_{\alpha} f, f^{\prime}\right]_{\mathcal{F}}=\left[f, D_{\alpha^{*}} f^{\prime}\right]_{\mathcal{F}}, \quad f, f^{\prime} \in \mathcal{F}, \alpha \in \Gamma .
$$

To see this, letting $f=\mathbf{T} g$ and $f^{\prime}=\mathbf{T} g^{\prime}$ for some $g, g^{\prime} \in \mathcal{G}$, we have

$$
\begin{aligned}
{\left[D_{\alpha} f, f^{\prime}\right]_{\mathcal{F}} } & =\sum_{\xi \in \Gamma}\left[f_{\alpha^{*} \xi}, g_{\xi}^{\prime}\right]_{\mathcal{H}} \\
& =\sum_{\xi \in \Gamma} \sum_{\eta \in \Gamma}\left[T_{\xi^{*} \alpha \eta} g_{\eta}, g_{\xi}^{\prime}\right]_{\mathcal{H}}
\end{aligned}
$$




$$
\begin{aligned}
& =\sum_{\xi \in \Gamma} \sum_{\eta \in \Gamma}\left[g_{\eta}, T_{\eta^{*} \alpha^{*} \xi} g_{\xi}^{\prime}\right]_{\mathcal{H}} \\
& =\sum_{\eta \in \Gamma}\left[g_{\eta}, f_{\alpha \eta}^{\prime}\right]_{\mathcal{H}}=\left[f, D_{\alpha^{*}} f^{\prime}\right]_{\mathcal{F}},
\end{aligned}
$$

where we also used Proposition 3.1, and hence the formula (3.22) is proven.

Observe that, so far, $D_{\xi}$ is only defined on $\mathcal{F}$. In order to show that $D_{\xi}$ extends from $\mathcal{F}$ to $\widehat{H}$, we have to show that $D$ exhibits the boundedness property. Indeed, letting $f \in \mathcal{F}$ such that $f=\mathbf{T} g$ for some $g \in \mathcal{G}$, by the definition of $D$ as in (3.19), condition (c), and formulae (3.21) and (3.22), we have

$$
\begin{aligned}
{\left[D_{\alpha} f, D_{\alpha} f\right]_{\mathcal{F}} } & =\left[D_{\alpha^{*}} D_{\alpha} f, f\right]_{\mathcal{F}}=\left[D_{\alpha^{*} \alpha} f, f\right]_{\mathcal{F}} \\
& =\sum_{\xi, \eta \in \Gamma}\left[T_{\xi^{*} \alpha^{*} \alpha \eta} g_{\eta}, g_{\xi}\right]_{\mathcal{H}} \\
& \leq c(\alpha)^{2} \sum_{\xi, \eta \in \Gamma}\left[T_{\xi^{*} \eta} g_{\eta}, g_{\xi}\right]_{\mathcal{H}} \\
& =c(\alpha)^{2}[f, f]_{\mathcal{F}},
\end{aligned}
$$

and the claim is proven. Since $D_{\xi^{*}}$ extends also by continuity and taking into account of (3.22), it follows that $D_{\xi^{*}}=D_{\xi}^{*}$, in particular, for any $\xi \in \Gamma$ the operator $D_{\xi}$ is adjointable, that is, $D_{\xi} \in \mathcal{B}^{*}(\widehat{\mathcal{H}})$.

Step 4. $T_{\xi}=V^{*} D_{\xi} V$ for all $\xi \in \Gamma$.

Taking into account of the definition of $V$ in (3.16), the definition of $D$ in (3.19), and the formula for calculating $V^{*}$ on $\mathcal{F}$ as in (3.18), for arbitrary $\xi \in \Gamma$, arbitrary $h \in \mathcal{H}$, and letting $\left\{g_{\eta}\right\}_{\eta \in \Gamma}=\left\{T_{\zeta^{*}} h\right\}_{\zeta \in \Gamma}$, that is, $g_{\eta}=T_{\zeta^{*}} h$ for $\eta=\zeta^{*}$, equivalently, $\eta^{*}=\zeta$, we have

$$
V^{*} D_{\xi} V h=V^{*} D_{\xi}\left\{T_{\zeta^{*}} h\right\}_{\zeta \in \Gamma}=V^{*}\left\{g_{\xi^{*} \eta}\right\}_{\eta \in \Gamma}=g_{\xi^{*}}=T_{\xi} h
$$

Step 5. $\overline{\operatorname{Lin}\left\{D_{\alpha} V \mathcal{H} \mid \alpha \in \Gamma\right\}}=\widehat{\mathcal{H}}$.

To this end, we prove that

$$
\operatorname{Lin}\left\{D_{\alpha} V \mathcal{H} \mid \alpha \in \Gamma\right\}=\mathcal{F}
$$

To see this, since the range of $V$ is included in $\mathcal{F}$ and $D_{\alpha}$ leaves $\mathcal{F}$ invariant, the direct inclusion holds. In order to prove the converse inclusion, let $f$ be arbitrary in $\mathcal{F}$, hence, there exists $g$ a finitely supported family of vectors in $\mathcal{H}$ such that $f=\mathbf{T} g$, that is, cf. (3.10),

$$
f=\left\{\sum_{\eta \in \Gamma} T_{\xi^{*} \eta} g_{\eta}\right\}_{\xi \in \Gamma}=\sum_{\eta \in \Gamma}\left\{T_{\xi^{*} \eta} g_{\eta}\right\}_{\xi \in \Gamma}=\sum_{\eta \in \Gamma} D_{\eta} V g_{\eta},
$$


where, at the last equality, we took into account of the definitions of $V$ as in (3.16) and of $D$ as in (3.19), in order to obtain $D_{\eta} V g_{\eta}=\left\{T_{\xi^{*} \eta} g_{\eta}\right\}_{\xi \in \Gamma}$, for all $\eta \in \Gamma$.

Since $\mathcal{F}$ is dense in $\widehat{\mathcal{H}}$, by construction, from (3.24) it follows the required conclusion.

Step 6. The uniqueness of $\widehat{\mathcal{H}}$.

If we have another VH-space $\widehat{\mathcal{H}}^{\prime}$, with corresponding $*$-representation $D: \Gamma \rightarrow$ $\mathcal{B}^{*}\left(\widehat{\mathcal{H}}^{\prime}\right)$, operator $V^{\prime} \in \mathcal{B}\left(\mathcal{H}, \widehat{\mathcal{H}}^{\prime}\right)$, such that $T_{\xi}=V^{\prime *} D_{\xi}^{\prime} V^{\prime}$, and minimality condition $\overline{\operatorname{Lin}\left\{D_{\alpha}^{\prime} V^{\prime} \mathcal{H} \mid \alpha \in \Gamma\right\}}=\widehat{\mathcal{H}}^{\prime}$, then for any finitely supported $h=\left\{h_{\alpha}\right\}_{\alpha \in \Gamma}$ and $k=\left\{k_{\beta}\right\}_{\beta \in \Gamma}$ we have

$$
\sum_{\alpha, \beta \in \Gamma}\left[D_{\alpha} V h_{\alpha}, D_{\beta} V k_{\beta}\right]_{\widehat{\mathcal{H}}}=\sum_{\alpha, \beta \in \Gamma}\left[T_{\beta^{*} \alpha} h_{\alpha}, k_{\beta}\right]_{\mathcal{H}}=\sum_{\alpha, \beta \in \Gamma}\left[D_{\alpha}^{\prime} V^{\prime} h_{\alpha}, D_{\beta}^{\prime} V^{\prime} k_{\beta}\right]_{\widehat{\mathcal{H}}^{\prime}}
$$

It follows that we can define an isometry $U$, on a dense linear subspace of $\widehat{\mathcal{H}}$ and valued in a dense linear subspace of $\widehat{\mathcal{H}}^{\prime}$, by

$$
\sum_{\alpha \in \Gamma} D_{\alpha} V f_{\alpha} \stackrel{U}{\longmapsto} \sum_{\alpha \in \Gamma} D_{\alpha}^{\prime} V^{\prime} f_{\alpha}
$$

which extends to an isomorphism of $\mathrm{VH}$-spaces

$$
U: \widehat{\mathcal{H}} \rightarrow \widehat{\mathcal{H}}^{\prime}
$$

Clearly, $U$ satisfies

$$
U D_{\xi}=D_{\xi}^{\prime} U \text { for all } \xi \in \Gamma
$$

We also observe that $D_{\epsilon}$ acts as the identity operator on $\mathcal{F}$ and hence, by the density of $\mathcal{F}$ in $\mathcal{H}$, it follows that $D_{\epsilon}=I_{\mathcal{H}}$. Similarly we have that $D_{\epsilon}^{\prime}=I_{\mathcal{H}}$. Therefore, by the definition of the operator $U$ it follows $U V=V^{\prime}$, hence $U$ has all the required properties.

If, in addition, we have that $T_{\epsilon}=I$ then $V^{*} V=V^{*} D_{\epsilon} V=T_{\epsilon}=I_{\mathcal{H}}$, that is, $V: \mathcal{H} \rightarrow \widehat{\mathcal{H}}$ is an isometry and then, identifying $\mathcal{H}$ with $V \mathcal{H}$, we have $T_{\xi}=P_{\mathcal{H}} D_{\xi} \mid \mathcal{H}$ for all $\xi \in \Gamma$.

$(2) \Rightarrow(1)$. With $(\widehat{\mathcal{H}} ; D ; V)$, a triple as in assertion $(2)$, for any finitely supported $g$ we have

$$
\begin{aligned}
\sum_{\xi, \eta \in \Gamma}\left[T_{\xi^{*} \eta} g_{\eta}, g_{\xi}\right]_{\mathcal{H}} & =\sum_{\xi, \eta \in \Gamma}\left[V^{*} D_{\xi^{*} \eta} V g_{\eta}, g_{\xi}\right]_{\widehat{\mathcal{H}}} \\
& =\sum_{\xi, \eta \in \Gamma}\left[D_{\xi^{*}} D_{\eta} V g_{\eta}, V g_{\xi}\right]_{\widehat{\mathcal{H}}}
\end{aligned}
$$




$$
\begin{aligned}
& =\sum_{\xi, \eta \in \Gamma}\left[D_{\eta} V g_{\eta}, D_{\xi} V g_{\xi}\right]_{\widehat{\mathcal{H}}} \\
& =\left[\sum_{\xi \in \Gamma} D_{\xi} V g_{\xi}, \sum_{\xi \in \Gamma} D_{\xi} V g_{\xi}\right]_{\widehat{\mathcal{H}}} \geq 0,
\end{aligned}
$$

hence $T$ is positive semidefinite. To verify the condition (c), we have

$$
\begin{aligned}
\sum_{\xi, \eta \in \Gamma}\left[T_{\xi^{*} \alpha^{*} \alpha \eta} f_{\eta}, f_{\xi}\right]_{\mathcal{H}} & =\sum_{\xi, \eta \in \Gamma}\left[V^{*} D_{\xi * \alpha^{*} \alpha \eta} V f_{\eta}, f_{\xi}\right]_{\mathcal{H}} \\
& =\sum_{\xi, \eta \in \Gamma}\left[D_{\alpha} D_{\eta} V f_{\eta}, D_{\alpha} D_{\xi} V f_{\xi}\right]_{\widehat{\mathcal{H}}} \\
& =\left[D_{\alpha} \sum_{\eta \in \Gamma} D_{\eta} V f_{\eta}, D_{\alpha} \sum_{\xi \in \Gamma} D_{\xi} V f_{\xi}\right]_{\widehat{\mathcal{H}}} \\
& \leq\left\|D_{\alpha}\right\|^{2}\left[\sum_{\eta \in \Gamma} D_{\eta} V f_{\eta}, \sum_{\xi \in \Gamma} D_{\xi} V f_{\xi}\right]_{\widehat{\mathcal{H}}} \\
& =\left\|D_{\alpha}\right\|^{2} \sum_{\xi, \eta \in \Gamma}\left[T_{\xi^{*} \eta} f_{\eta}, f_{\xi}\right]_{\mathcal{H}},
\end{aligned}
$$

and the proof is finished.

The next corollary shows that the construction provided by the previous theorem carries over to the case when some linearity properties occur, cf. [14,27].

Corollary 3.4 With the notation as in Theorem 3.3, if we assume, in addition to the assumptions in the assertion (1), that $T_{\xi \alpha \eta}=T_{\xi \beta \eta}+T_{\xi \gamma \eta}$ for some fixed $\alpha, \beta, \gamma$ and all $\xi, \eta$ in $\Gamma$, then $D_{\alpha}=D_{\beta}+D_{\gamma}$.

Proof We use the notation as in the proof of Theorem 3.3. We know that any element $f \in \mathcal{F}$ is of the form $f=\mathbf{T} g$ for some $g \in \mathcal{G}$, hence

$$
f_{\xi}=\sum_{\eta \in \Gamma} T_{\xi^{*} \eta} g_{\eta}=\sum_{\eta \in \Gamma} D_{\xi}\left(T_{\eta} g_{\eta}\right)=D_{\xi} \sum_{\eta \in \Gamma} T_{\eta} g_{\eta}, \quad \xi \in \Gamma .
$$

So, from the assumption $T_{\xi \alpha \eta}=T_{\xi \beta \eta}+T_{\xi \gamma \eta}$ it follows that $D_{\alpha^{*} \xi^{*}}=D_{\beta^{*} \xi^{*}}+$ $D_{\gamma^{*} \xi^{*}}$. Since $T_{\xi}=V^{*} D_{\xi} V$ and using the minimality condition, from here it follows that $T_{\alpha^{*} \xi^{*}}=T_{\beta^{*} \xi^{*}}+T_{\gamma^{*} \xi^{*}}$. Finally, using the observation that the collection of all $\left\{T_{\xi^{*}} g_{\xi^{*}}\right\}_{\xi \in \Gamma}$, when $g \in \mathcal{G}$, also spans $\mathcal{F}$, from here we obtain $D_{\alpha}=D_{\beta}+D_{\gamma}$.

\section{Stinespring's Theorem for VH-Spaces}

In this section we investigate Stinespring's type theorems for $\mathcal{B}^{*}(\mathcal{H})$-valued maps, in connection to the generalized Sz.-Nagy's Theorem. 


\section{$4.1 \mathcal{B}^{*}(\mathcal{H})$-Valued Maps on $*$-algebras}

Let $\mathcal{A}$ be a complex $*$-algebra with unit 1 . Recall that the involution $*$ is supposed to be conjugate linear, $(a b)^{*}=b^{*} a^{*}$ for all $a, b \in \mathcal{A},\left(a^{*}\right)^{*}=a$ for all $a \in \mathcal{A}$, and that $1^{*}=1$. In particular, $\mathcal{A}$ has an underlying structure of a unital multiplicative $*$-semigroup. For an arbitrary $\mathrm{VH}$-space $\mathcal{H}$ over the admissible space $Z$, let $\varphi: \mathcal{A} \rightarrow \mathcal{B}^{*}(\mathcal{H})$ be a linear map. With this observation, the notions of different types of positivity introduced in Sect. 3 have immediate transcription to this setting: $\varphi$ is $n$-positive, for some natural number $n$, if for any $a_{1}, \ldots, a_{n} \in \mathcal{A}$ and $h_{1}, \ldots, h_{n} \in \mathcal{H}$ we have

$$
\sum_{i, j=1}^{n}\left[\varphi\left(a_{i}^{*} a_{j}\right) h_{j}, h_{i}\right]_{\mathcal{H}} \geq 0,
$$

and, respectively, $\varphi$ is positive semidefinite if it is $n$-positive for all $n \in \mathbb{N}$.

There is another notion of positivity that has been considered, following the original terminology of Stinespring [24]. Given $\mathcal{A}$ a $*$-algebra, a linear map $\varphi: \mathcal{A} \rightarrow \mathcal{B}^{*}(\mathcal{H})$ is called positive if $\varphi\left(a^{*} a\right) \geq 0$ for any $a \in \mathcal{A}$. Given $n \in \mathbb{N}$, there is a natural identification of $*$-algebras of $M_{n}(\mathcal{A})$, the algebra of all $n \times n$ matrices with entries in $\mathcal{A}$, with $M_{n} \otimes \mathcal{A}$, organized as a $*$-algebra similarly in a natural way (e.g. see [21]). A linear map $\varphi_{n}: M_{n}(\mathcal{A}) \rightarrow M_{n}\left(\mathcal{B}^{*}(\mathcal{H})\right)$ is naturally associated to $\varphi$ by

$$
\varphi_{n}\left(\left[a_{i, j}\right]_{i, j=1}^{n}\right)=\left[\varphi\left(a_{i, j}\right)\right]_{i, j=1}^{n}, \quad\left[a_{i, j}\right]_{i, j=1}^{n} \in M_{n}(\mathcal{A}) .
$$

The importance of this notion relies on its "quantization" interpretation, e.g. see [7].

Taking into account that any positive element $A=\left[a_{i, j}\right]_{i, j=1}^{n} \in M_{n}(\mathcal{A})$ can be decomposed

$$
A=A_{1}^{*} A_{1}+\cdots+A_{n}^{*} A_{n}
$$

where $A_{k}$ is the $n \times n$ matrix having its $k$-th row with entries $a_{k, j}, j=1, \ldots, n$, and all the other entries null, we get the following fact, essentially proven in [24]:

Proposition 4.1 Let $\mathcal{A}$ be a *-algebra, $\mathcal{H}$ a VH-space, and a linear map $\varphi: \mathcal{A} \rightarrow$ $\mathcal{B}^{*}(\mathcal{H})$.

(a) For arbitrary $n \in \mathbb{N}$, $\varphi$ is n-positive if and only if $\varphi_{n}$ is positive.

(b) $\varphi$ is positive semidefinite if and only if $\varphi_{n}$ is positive for all $n \in \mathbb{N}$

$\varphi$ is called completely positive if $\varphi_{n}$ is positive for all $n \in \mathbb{N}$, hence the statement (b) says that complete positivity is the same with positive semidefiniteness, in this setting.

The preliminary results on $\mathcal{B}^{*}(\mathcal{H})$-valued maps as in Subsection 3.1 have counterparts in this setting. Thus, by Proposition 3.1 and Lemma 3.2 we have:

Lemma 4.2 Let $\mathcal{A}$ be a complex unital $*$-algebra and $\varphi: \mathcal{A} \rightarrow \mathcal{B}^{*}(\mathcal{H})$, for some $V H$-space $\mathcal{H}$. If $\varphi$ is at least 2-positive then it is selfadjoint in the sense that $\varphi\left(a^{*}\right)=$ $\varphi(a)^{*}$ for all $a \in \mathcal{A}$. 
On the other hand, as a consequence of Theorem 3.3 and its Corollary 3.4, we have:

Corollary 4.3 Let $\mathcal{A}$ be a complex $*$-algebra and $\mathcal{H}$ a VH-space over an admissible space Z. Given a linear map $\varphi: \mathcal{A} \rightarrow \mathcal{B}^{*}(\mathcal{H})$, the following assertions are equivalent:

(i) $\varphi$ has the properties:

(b) $\varphi$ is completely positive on $\mathcal{A}$.

(c) For any $a \in \mathcal{A}$ there exists a nonnegative number $c(a)$ such that

$$
\sum_{b, c \in \mathcal{A}}\left[\varphi\left(c^{*} a^{*} a b\right) h_{b}, h_{c}\right]_{\mathcal{H}} \leq c(a)^{2} \sum_{b, c \in \mathcal{A}}\left[\varphi\left(c^{*} b\right) h_{b}, h_{c}\right]_{\mathcal{H}},
$$

for all finitely supported families $\left\{h_{b}\right\}_{b \in \mathcal{A}}$ of vectors in $\mathcal{H}$.

(ii) There exists a triple $(\mathcal{K} ; \Phi ; V)$, consisting of a $V H$-space $\mathcal{K}$ over the same admissible space $Z$, an operator $V \in \mathcal{B}^{*}(\mathcal{H}, \mathcal{K})$, and a $*$-representation $\Phi: \mathcal{A} \rightarrow \mathcal{B}^{*}(\mathcal{K})$ of $*$ - algebras, such that $\varphi(a)=V^{*} \Phi(a) V$, for all $a \in \mathcal{A}$.

\subsection{A Generalized Stinespring's Theorem}

The classical Stinespring's Theorem [24] says that, for the case of a Hilbert space $\mathcal{H}$ and $C^{*}$-algebra $\mathcal{A}$, condition (c) in Corollary 4.3 is not necessary. An enhanced version of Stinespring's Theorem, e.g. see [2], states that this is true when $\mathcal{A}$ is a $B^{*}$-algebra as well. It is our aim to show that this holds for the case of a VH-space. We first get this generalization of the Stinespring's Theorem as a consequence of the generalized Sz.-Nagy's Theorem 3.3, then we present a direct proof, based on the original ideas in [24].

Theorem 4.4 Let $\mathcal{A}$ be a unital $B^{*}$-algebra, $\mathcal{H}$ a $V H$-space over an admissible space $Z$, and let $\varphi: \mathcal{A} \rightarrow \mathcal{B}^{*}(H)$ be a linear map. Then $\varphi$ is positive semidefinite if and only if there exists $\mathcal{K}$ a VH-space over the same admissible space $Z$, an operator $V \in \mathcal{B}^{*}(\mathcal{H}, \mathcal{K})$ and $a *$-representation $\rho: \mathcal{A} \rightarrow \mathcal{B}^{*}(\mathcal{K})$ such that

$$
\varphi(a)=V^{*} \rho(a) V, \text { for all } a \in \mathcal{A} .
$$

Moreover, the VH-space $\mathcal{K}$ can be obtained minimal, that is, $\mathcal{K}=\overline{\operatorname{Lin}\{\varphi(\mathcal{A}) \mathcal{H}\}}$, and in this case, the triple $(\rho ; V ; \mathcal{K})$ is unique, modulo a unitary operator of VH-spaces that intertwines the $*$-representations and keeps the operators $V$.

In addition, if $\varphi$ is unital, $\mathcal{H}$ can be isometrically embedded as an orthocomplemented subspace of $\mathcal{K}$ and, letting $P_{\mathcal{H}}$ denote the orthogonal projection onto $\mathcal{H}$, we have

$$
\varphi(a)=\left.P_{\mathcal{H}} \rho(a)\right|_{\mathcal{H}}, \text { for all } a \in \mathcal{A} \text {. }
$$

First Proof As a consequence of Theorem 3.3 and its Corollary 4.3, we only have to prove that, assuming the $\varphi$ is positive semidefinite, then condition (c) in Corollary 4.3 
holds automatically. To this end, let $a \in \mathcal{A}$ and finitely supported $\left\{h_{b}\right\}_{b \in \mathcal{A}}$ in $\mathcal{H}$. Since $\varphi$ is positive semidefinite, for any $y \in \mathcal{A}$ we have

$$
\sum_{b, c \in \mathcal{A}}\left[\varphi\left(c^{*} y^{*} y b\right) h_{b}, h_{c}\right]_{\mathcal{H}} \geq 0
$$

Without loss of generality we can assume that $\|a\|<1$ and let $x=a^{*} a$, hence $\|x\| \leq\|a\|^{2}<1$. Following an idea in [2] and using an exercise in [1] at page 125, we consider the power series of the analytic complex function $(1-\lambda)^{1 / 2}$ that converges in the open unit disc

$$
(1-\lambda)^{1 / 2}=1-\sum_{n \geq 1} c_{n} \lambda^{n}
$$

and let

$$
y=1-\sum_{n \geq 1} c_{n} x^{n} \in \mathcal{A} .
$$

It is easy to see that $y=y^{*}$, since $x=x^{*}$, and that $1-a^{*} a=1-x=y^{2}$, hence, from (4.5) it follows

$$
\sum_{b, c \in \mathcal{A}}\left[\varphi\left(c^{*} a^{*} a b\right) h_{b}, h_{c}\right]_{\mathcal{H}} \leq \sum_{b, c \in \mathcal{A}}\left[\varphi\left(c^{*} b\right) h_{b}, h_{c}\right]_{\mathcal{H}}
$$

which proves (4.2).

Second Proof We only prove the direct implication; the converse one shows no obstruction. Also, since most of the arguments are similar with the original proof in [24], we focus mainly on the obstructions coming from the VH-space anomalies. Thus, assume that $\varphi$ is positive semidefinite and consider the algebraic tensor product $\mathcal{A} \otimes \mathcal{H}$ on which a $Z$-valued pairing $[\cdot, \cdot]$ is defined as follows:

$$
\left[\sum_{j=1}^{n} a_{j} \otimes x_{j}, \sum_{k=1}^{m} b_{k} \otimes y_{k}\right]:=\sum_{j=1}^{n} \sum_{k=1}^{m}\left[\varphi\left(b_{k}^{*} a_{j}\right) x_{j}, y_{k}\right]_{\mathcal{H}},
$$

for any $a_{1}, \ldots, a_{n}, b_{1}, \ldots, b_{m} \in \mathcal{A}$ and $x_{1}, \ldots, x_{n}, y_{1}, \ldots, y_{m} \in \mathcal{H}$. First we have to note that this definition is correct (the representations as sums of elementary tensors in $\mathcal{A} \otimes \mathcal{H}$ are not unique). Then, we show that this pairing satisfies all the axioms of a $Z$-valued inner product, but the definiteness. By Lemma 2.3 it follows that

$\mathcal{N}:=\{f \in \mathcal{A} \otimes \mathcal{N} \mid[f, f]=0\}=\left\{f \in \mathcal{A} \otimes \mathcal{N} \mid\left[f, f^{\prime}\right]=0\right.$ for all $\left.f^{\prime} \in \mathcal{A} \otimes \mathcal{H}\right\}$

For this reason, we first have to factor to a VE-space $(\mathcal{A} \otimes \mathcal{H}) / \mathcal{N}$ and then take $\mathcal{K}$ its completion to a VH-space. 
A *-representation of $\mathcal{A}$ on $\mathcal{A} \otimes \mathcal{H}$ is defined in the usual way:

$$
\rho(a)\left(\sum_{j=1}^{n} a_{j} \otimes x_{j}\right):=\sum_{j=1}^{n} a a_{j} \otimes x_{j}, \quad a \in \mathcal{A} .
$$

We obtain the following inequality

$$
[\rho(a) f, \rho(a) f] \leq 2\left\|a^{*} a\right\|[f, f], \quad a \in \mathcal{A}, f \in \mathcal{A} \otimes \mathcal{H},
$$

using the same arguments as those for the proof of (4.7). This shows that $\rho(a)$ factors and extends by continuity to a bounded operator in $\mathcal{K}$. Since $\rho$ is formally selfadjoint, it follows that $\rho(a) \in \mathcal{B}^{*}(\mathcal{K})$ and $\rho\left(a^{*}\right)=\rho(a)^{*}$ for all $a \in \mathcal{A}$. Thus, $\rho: \mathcal{A} \rightarrow \mathcal{B}^{*}(\mathcal{K})$ is a $*$-representation.

The operator $V$ is defined by $V x:=1 \otimes x+\mathcal{N} \in(\mathcal{A} \otimes \mathcal{H}) / \mathcal{N}$, for all $x \in \mathcal{H}$. We first have to show that $V$ is bounded. To see this, we first note that from $[f-g, f-g] \geq 0$ it follows that $[f, g]+[g, f] \leq[f, f]+[g, g]$ for arbitrary $f, g \in \mathcal{A} \otimes \mathcal{H}$, hence

$$
\begin{aligned}
{[V x, V x] } & =[1 \otimes x, 1 \otimes x]=[\varphi(1) x, x] \\
& \leq \frac{1}{2}([\varphi(1) x, x]+[x, \varphi(1) x]) \\
& \leq \frac{1}{2}([\varphi(1) x, \varphi(1) x]+[x, x]) \\
& \leq \frac{\|\varphi(1)\|^{2}+1}{2}[x, x] .
\end{aligned}
$$

We thus can extend $V$ to a bounded operator $\mathcal{H} \rightarrow \mathcal{K}$.

On the other hand, from

$$
\begin{aligned}
{[V x, a \otimes y] } & =[1 \otimes x, a \otimes y]=\left[\varphi\left(a^{*}\right) x, y\right]_{\mathcal{H}}=\left[x, \varphi\left(a^{*}\right)^{*} y\right]_{\mathcal{H}} \\
& =[x, \varphi(a) y]_{\mathcal{H}}, \quad x, y \in \mathcal{H}, a \in \mathcal{A},
\end{aligned}
$$

where the last equality is due to Lemma 4.2, it follows that we can use Lemma 2.4 and conclude that $V$ is adjointable and that $V^{*}(a \otimes y)=\varphi(a) y$ for all $a \in \mathcal{A}$ and all $y \in \mathcal{H}$.

Finally,

$$
\begin{aligned}
{\left[V^{*} \rho(a) V x, y\right]_{\mathcal{H}} } & =[\rho(a) V x, V y] \\
& =[\rho(a)(1 \otimes x),(1 \otimes y)] \\
& =[a \otimes x, 1 \otimes y] \\
& =[\varphi(a) x, y]_{\mathcal{H}}, \quad x, y \in \mathcal{H}, a \in \mathcal{A},
\end{aligned}
$$

hence $\varphi(a)=V^{*} \rho(a) V$ for all $a \in \mathcal{A}$.

All the other assertions are clear now. 
4.3 Equivalence of the Generalized Sz.-Nagy and Stinespring Theorems for VH-Spaces

In the following we prove that Szafraniec's theorem [26] holds in the case of $\mathcal{B}^{*}(\mathcal{H})$ valued maps, for $\mathcal{H}$ a VH-space, as well. To a large extent we use the original idea in [26]: first choose conveniently the constants $c$ in the boundedness condition (this was originally done in [25], but in our case those proofs do not work entirely) and then linearize the map to a weighted $\ell_{c}^{1}$ space that can be shown to have all the necessary properties in order to make it a $B^{*}$-algebra but, apart of the anomalies of $\mathrm{VH}$-spaces that we have to deal with on the way, we take advantage of the fact that the generalized Stinespring's Theorem is obtained for the more general $B^{*}$-algebras, instead of $C^{*}$-algebras, and hence avoid the complications of enveloping.

Theorem 4.5 The generalized Sz.-Nagy's Dilation Theorem 3.3 is logically equivalent with the generalized Stinespring's Dilation Theorem 4.4.

Proof We already got Theorem 4.4 by Theorem 3.3, so we only have to prove the converse implication. To this end, we assume now that Theorem 4.4 is known, consider $\Gamma$ a unital $*$-semigroup, and let $T: \Gamma \rightarrow \mathcal{B}^{*}(\mathcal{H})$ for some VH-space $\mathcal{H}$. The statement of Theorem 3.3 is an equivalence, in which one implication does not show any difficulty. So, it remains to show that the implication $(1) \Rightarrow(2)$ in Theorem 3.3 can be put within the framework of Theorem 4.4. To this end, let us assume that $T$ is positive semidefinite and satisfies the boundedness condition (c), as in Theorem 3.3. We divide the proof in three steps:

Step 1. There exists a mapping $c: \Gamma \rightarrow[1,+\infty)$, subject to the following properties:

(a) $c$ is submultiplicative, that is, $c(\alpha \beta) \leq c(\alpha) c(\beta)$ for all $\alpha, \beta \in \Gamma$.

(b) There exists $C>0$ such that $\left\|T_{\alpha}\right\| \leq C c(\alpha)$ for all $\alpha \in \Gamma$.

(c) For all $\alpha \in \Gamma, c(\alpha)$ satisfies the boundedness condition (3.5).

(d) $c\left(\alpha^{*}\right)=c(\alpha)$ for all $\alpha \in \Gamma$.

(e) $c(\epsilon)=1$.

Indeed, for each $\alpha \in \Gamma$ let

$$
\begin{aligned}
& c(\alpha)=\inf \left\{c \geq 0 \mid \sum_{\xi, \eta \in \Gamma}\left[T_{\xi^{*} \alpha^{*} \alpha \eta} g_{\eta}, g_{\xi}\right]_{\mathcal{H}} \leq c^{2} \sum_{\xi, \eta \in \Gamma}\left[T_{\xi^{*} \eta} g_{\eta}, g_{\xi}\right]_{\mathcal{H}}\right. \\
& \text { holds for all } \mathcal{H} \text {-valued finitely supported family } g\} \text {. }
\end{aligned}
$$

Actually, $c(\alpha)$ is the minimum (that is, the infimum is attained) since the set from which we take the infimum is closed. Let $\alpha, \beta \in \Gamma, n \in \mathbb{N}, h_{1}, \ldots, h_{n} \in \mathcal{H}$, and 
$\xi_{1}, \ldots, \xi_{n} \in \Gamma$. Then

$$
\begin{aligned}
& \sum_{j, k=1}^{n}\left[T_{\xi_{k}^{*} \beta^{*} \alpha^{*} \alpha \beta \xi_{j}} h_{j}, h_{k}\right]_{\mathcal{H}} \leq c(\alpha) \sum_{j, k=1}^{n}\left[T_{\xi_{k}^{*} \beta^{*} \beta \xi_{j}} h_{j}, h_{k}\right]_{\mathcal{H}} \\
& \leq c(\alpha) c(\beta) \sum_{j, k=1}^{n}\left[T_{\xi_{k}^{*} \xi_{j}} h_{j}, h_{k}\right]_{\mathcal{H}},
\end{aligned}
$$

hence $c(\alpha \beta) \leq c(\alpha) c(\beta)$.

Let us fix $\alpha \in \Gamma$. By (3.5) we have

$$
\left[T_{\alpha^{*} \alpha} h, h\right]_{\mathcal{H}} \leq c(\alpha)^{2}\left[T_{\epsilon} h, h\right]_{\mathcal{H}} \leq c(\alpha)^{2}\left\|T_{\epsilon}\right\|[h, h]_{\mathcal{H}} \text {, for all } h \in \mathcal{H}
$$

Then, letting $f=\mathbf{T} \delta_{\alpha} h$ for arbitrary $h \in \mathcal{H}$ and taking into account that the operator $V^{*}$ as in (3.18) is bounded and that $\left\|V^{*}\right\|=\|V\|=\left\|T_{\epsilon}\right\|$, it follows

$$
\begin{aligned}
{\left[T_{\alpha} h, T_{\alpha} h\right]_{\mathcal{H}} } & =\left[f_{\epsilon}, f_{\epsilon}\right]_{\mathcal{H}}=\left[V^{*} h, V^{*} h\right]_{\mathcal{H}} \\
& \leq\left\|T_{\epsilon}\right\|[f, f]_{\mathcal{F}}=\left\|T_{\epsilon}\right\|\left[T_{\alpha^{*} \alpha} h, h\right]_{\mathcal{H}} \leq c(\alpha)^{2}\left\|T_{\epsilon}\right\|^{2}[h, h]_{\mathcal{H}} .
\end{aligned}
$$

Therefore,

$$
\left\|T_{\alpha}\right\| \leq\left\|T_{\epsilon}\right\| c(\alpha)
$$

Note that $c(\epsilon)=1$ holds, by definition.

In the following we prove that $c\left(\alpha^{*}\right)=c(\alpha)$ for all $\alpha \in \Gamma$. To see this, and in order to emphasize the ideas and simplify the calculations, we use the construction in Step 3 of the proof of Theorem 3.3. More precisely, we consider the linear operators $D_{\alpha}: \mathcal{F} \rightarrow \mathcal{F}$ as in (3.19) and use the fact, already established in (3.23), that

(3.5) holds if and only if $\left[D_{\alpha} f, D_{\alpha} f\right]_{\mathcal{F}} \leq c(\alpha)^{2}[f, f]_{\mathcal{F}}$, for all $f \in \mathcal{F}$.

Let $\alpha \in \Gamma$ be fixed and assume that $c(\alpha) \leq 1$. We now follow an idea from [14]. Writing down that $[u-v, u-v]_{\mathcal{F}} \geq 0$ it follows that $[u, v]_{\mathcal{F}}+[v, u]_{\mathcal{F}} \leq[u, u]_{\mathcal{F}}+[v, v]_{\mathcal{F}}$. Then, using (3.22), for any $f \in \mathcal{F}$ we have

$$
\begin{aligned}
{\left[D_{\alpha^{*}} f, D_{\alpha^{*}} f\right]_{\mathcal{F}} } & =\frac{1}{2}\left(\left[D_{\alpha^{*}} f, D_{\alpha^{*}} f\right]_{\mathcal{F}}+\left[D_{\alpha^{*}} f, D_{\alpha^{*}} f\right]_{\mathcal{F}}\right) \\
& =\frac{1}{2}\left(\left[D_{\alpha} D_{\alpha^{*}} f, f\right]_{\mathcal{F}}+\left[f, D_{\alpha} D_{\alpha^{*}} f\right]_{\mathcal{F}}\right) \\
& \leq \frac{1}{2}\left(\left[D_{\alpha} D_{\alpha^{*}} f, D_{\alpha} D_{\alpha^{*}} f\right]_{\mathcal{F}}+[f, f]_{\mathcal{F}}\right) \\
& \leq \frac{1}{2}\left(\left[D_{\alpha^{*}} f, D_{\alpha^{*}} f\right]_{\mathcal{F}}+[f, f]_{\mathcal{F}}\right),
\end{aligned}
$$

hence $\left[D_{\alpha^{*}} f, D_{\alpha^{*}} f\right]_{\mathcal{F}} \leq[f, f]_{\mathcal{F}}$, which, via (4.11), implies that $c\left(\alpha^{*}\right) \leq 1$. Thus, we proved that $c\left(\alpha^{*}\right) \leq c(\alpha)$ and hence, by symmetry $c\left(\alpha^{*}\right)=c(\alpha)$ for all $\alpha \in \Gamma$. 
Finally, it is easy to see that, replacing $c(\alpha)$ by $\max \{c(\alpha), 1\}$, all the properties (a)-(e) are preserved.

Step 2. The unital $B^{*}$-algebra $\ell_{c}^{1}(\Gamma)$.

Let $\ell_{c}^{1}(\Gamma)$ be the set of all $f: \Gamma \rightarrow \mathbb{C}$ such that

$$
\|f\|_{c}:=\sum_{\gamma \in \Gamma} c(\gamma)\left|f_{\gamma}\right|<\infty
$$

For the moment $\ell_{c}^{1}(\Gamma)$ is a complex vector space and $\|f\|_{c}$ is a complete norm on it. A multiplication in $\ell_{c}^{1}(\Gamma)$ can be defined by the convolution: given $f, g \in \ell_{c}^{1}(\Gamma)$ we let $f * g: \Gamma \rightarrow \mathbb{C}$ be defined by

$$
(f * g)_{\xi}=\sum_{\eta \gamma=\xi} f_{\eta} g_{\gamma}, \quad \xi \in \Gamma .
$$

Then

$$
\begin{aligned}
\|f * g\|_{c} & =\sum_{\xi \in \Gamma} c(\xi)\left|(f * g)_{\xi}\right| \\
& \leq \sum_{\xi \in \Gamma} c(\xi) \sum_{\eta \gamma=\xi}\left|f_{\eta} g_{\gamma}\right| \\
& =\sum_{\xi \in \Gamma} \sum_{\eta \gamma=\xi} c(\eta \gamma)\left|f_{\eta} g_{\gamma}\right| \\
& \leq \sum_{\eta, \gamma \in \Gamma} c(\eta) c(\gamma)\left|f_{\eta}\right|\left|g_{\gamma}\right| \\
& =\left(\sum_{\eta \in \Gamma} c(\eta)\left|f_{\eta}\right|\right)\left(\sum_{\gamma \in \Gamma} c(\gamma)\left|g_{\gamma}\right|\right)=\|f\|_{c}\|g\|_{c}
\end{aligned}
$$

hence the sum in (4.13) is absolutely convergent, $f * g \in \ell_{c}^{1}(\Gamma)$ and, with respect to this multiplication, $\ell_{c}^{1}(\Gamma)$ is a Banach algebra with unit $\delta_{\epsilon}$.

An involution on $\ell_{c}^{1}(\Gamma)$ is defined by complex conjugation and involution on $\Gamma$ : $f_{\xi}^{*}=\overline{f_{\xi^{*}}}$ for all $\xi \in \Gamma$. The involution $*$ is isometric with respect to the norm $\|\cdot\|_{c}$ :

$$
\left\|f^{*}\right\|_{c}=\sum_{\xi \in \Gamma} c(\xi)\left|f_{\xi}^{*}\right|=\sum_{\xi \in \Gamma} c(\xi)\left|f_{\xi^{*}}\right|=\sum_{\xi \in \Gamma} c\left(\xi^{*}\right)\left|f_{\xi^{*}}\right|=\sum_{\eta \in \Gamma} c(\eta)\left|f_{\eta}\right|=\|f\|_{c} .
$$

Thus, $\ell_{c}^{1}(\Gamma)$ is a unital $B^{*}$-algebra.

Step 3. The linearization of $T$ to the $B^{*}$-algebra $\ell_{c}^{1}(\Gamma)$. 
We define, and denote by the same letter $T$, a map $T: \ell_{c}^{1}(\Gamma) \rightarrow \mathcal{B}^{*}(\mathcal{H})$ by

$$
T f:=\sum_{\gamma \in \Gamma} f_{\gamma} T_{\gamma}, \quad f \in \ell_{c}^{1}(\Gamma)
$$

with the observation that the sum is absolutely convergent in $\mathcal{B}^{*}(\mathcal{H})$ since

$$
\sum_{\gamma \in \Gamma}\left|f_{\gamma}\right|\left\|T_{\gamma}\right\| \leq C \sum_{\gamma \in \Gamma}\left|f_{\gamma}\right| c(\gamma)=C\|f\|_{c}<\infty, \quad f \in \ell_{c}^{1}(\Gamma) .
$$

Clearly, $T$ is linear and, as a consequence of the previous inequality, we have

$$
\|T f\| \leq C\|f\|_{c}, \quad f \in \ell_{c}^{1}(\Gamma)
$$

hence it is bounded. Note that $T_{\eta}=T \delta_{\eta}$ for all $\eta \in \Gamma$, and we call $T$ the linearization to the $B^{*}$-algebra $\ell_{c}^{1}(\Gamma)$.

In the following we prove that $T$ is positive semidefinite on $\ell_{c}^{1}(\Gamma)$ as well. To see this, let $f_{1}, \ldots, f_{n} \in \ell_{c}^{1}(\Gamma)$ be finitely supported and $h_{1}, \ldots, h_{n} \in \mathcal{H}$ be arbitrary. Then, by (4.13), and (4.14), we have

$$
\begin{aligned}
\sum_{j, k=1}^{n}\left[T\left(f_{k}^{*} * f_{j}\right) h_{j}, h_{k}\right]_{\mathcal{H}} & =\sum_{j, k=1}^{n} \sum_{\xi \in \Gamma}\left[\left(f_{k}^{*} * f_{j}\right)_{\xi} T_{\xi} h_{j}, h_{k}\right]_{\mathcal{H}} \\
& =\sum_{j, k=1}^{n} \sum_{\xi \in \Gamma} \sum_{\eta \gamma=\xi}\left[\left(f_{k}^{*}\right)_{\eta}\left(f_{j}\right)_{\gamma} T_{\xi} h_{j}, h_{k}\right]_{\mathcal{H}} \\
& =\sum_{j, k=1}^{n} \sum_{\eta, \gamma \in \Gamma}\left[\overline{\left(f_{k}\right)_{\gamma^{*}}}\left(f_{j}\right)_{\eta} T_{\gamma \eta} h_{j}, h_{k}\right]_{\mathcal{H}}
\end{aligned}
$$

and then changing the index $\gamma$ to $\gamma^{*}$ and interchanging the sums, since they have only finitely many terms, we get

$$
=\sum_{\eta, \gamma \in \Gamma}\left[T_{\gamma^{*} \eta}\left(\sum_{j=1}^{n}\left(f_{j}\right)_{\eta} h_{j}\right),\left(\sum_{k=1}^{n}\left(f_{k}\right)_{\gamma} h_{k}\right)\right]_{\mathcal{H}} \geq 0,
$$

since $T$ is positive semidefinite on $\Gamma$. This calculation is sufficient to prove that $T$ is positive semidefinite on $\ell_{c}^{1}(\Gamma)$ since, by (4.15) and the fact that the linear manifold of all finitely supported complex functions on $\Gamma$ is a dense linear manifold in $\ell_{c}^{1}(\Gamma)$, a standard approximation procedure can be performed.

Finally, we apply the generalized Stinespring Theorem 4.4 to the positive semidefinite map $T: \ell_{c}^{1}(\Gamma) \rightarrow \mathcal{B}^{*}(\mathcal{H})$ and get a triple $(\rho ; V ; \mathcal{K})$, where $\mathcal{K}$ is a VH-space over the same admissible space $Z, V \in \mathcal{B}^{*}(\mathcal{H}, \mathcal{K})$, and $\rho: \ell_{c}^{1}(\Gamma) \rightarrow \mathcal{B}^{*}(\mathcal{K})$ is a *-representation, such that $T f=V^{*} \rho(f) V$ for all $f \in \ell_{c}^{1}(\Gamma)$. Letting $\widehat{\mathcal{H}}:=\mathcal{K}$ and 
$D_{\xi}=\rho(\delta \xi)$ we obtain a triple $(\widehat{\mathcal{H}} ; V ; D)$ that satisfies all the requirements as in assertion (2) in Theorem 3.3.

\section{References}

1. Arveson, W.A.: A Short Course on Spectral Theory, Graduate Texts in Mathematics, vol. 209. SpringerVerlag, Berlin (2002)

2. Arveson, W.B.: Dilation theory yesterday and today. In: A Glimpse at Hilbert Space Operators, pp. 99-123. Oper. Theory Adv. Appl., vol. 207, Birkhäuser verlag, Basel 2010

3. Chobanyan, S.A., Weron, A.: Banach-space-valued stationary processes and their linear prediction. Diss. Math. 125, 1-45 (1975)

4. Conway, J.B.: A Course in Operator Theory, Graduate Studies in Mathematics, vol. 21. American Mathematical Society, Providence (2000)

5. Davies, E.B.: Quantum Theory of Open Systems. Academic Press [Harcourt Brace Jovanovich, Publishers], London (1976)

6. Dixmier, J.: $C^{*}$-Algebras, North-Holland Mathematical Library, vol. 15. North-Holland Publishing Co, Amsterdam (1977)

7. Effros, E., Ruan, Zh.-J.: Operator Spaces. Oxford University Press, Oxford (2000)

8. Gaşpar, D., Gaşpar, P.: An operatorial model for Hilbert $\mathcal{B}(X)$-modules. Analele Univ. de Vest Timişoara Ser. Mat.-Inform. 40, 15-29 (2002)

9. Hayashi, M.: Quantum Information. An Introduction. Springer, Berlin (2006)

10. Inoue, A.: Locally $C^{*}$-algebras. Mem. Fac. Sci. Kyushu Univ. Ser. A 25, 197-235 (1971)

11. Kaplansky, I.: Modules over operator algebras. Am. J. Math. 75, 839-853 (1953)

12. Kasparov, G.G.: Hilbert $C^{*}$-modules: theorems of Stinespring and Voiculescu. J. Oper. Theory 4, $133-150(1980)$

13. Lance, E.C.: Hilbert $C^{*}$-Modules. A toolkit for operator algebraists. In: London Mathematical Society Lecture Note Series, 210. Cambridge University Press, Cambridge (1995)

14. Loynes, R.M.: On generalized positive-definite function. Proc. London Math. Soc. III. Ser. 15, 373-384 (1965)

15. Loynes, R.M.: Linear operators in $V H$-spaces. Trans. Amer. Math. Soc. 116, 167-180 (1965)

16. Loynes, R.M.: Some problems arising from spectral analysis. In: Symposium on Probability Methods in Analysis (Loutraki, 1966), pp. 197-207. Springer, Berlin (1967)

17. Manuilov V., Troitsky E.: Hilbert $C^{*}$ - Modules. American Mathematical Society, Providence (2005)

18. NaĬmark, M.A.: On the representations of additive operator set functions. C.R. (Doklady) Acad. Sci. USSR 41, 359-361 (1943)

19. Nă̌mark, M.A.: Positive definite operator functions on a commutative group. Bull. (Izv.) Acad. Sci. USSR 7, 237-244 (1943)

20. Paschke, W.L.: Inner product modules over $B^{*}$-algebras. Trans. Amer. Math. Soc. 182, 443-468 (1973)

21. Paulsen, V.R.: Completely Bounded Maps and Operator Algebras. Cambridge University Press, Cambridge (2002)

22. Phillips, N.C.: Inverse limits of $C^{*}$-algebras. J. Oper. Theory 19, 159-195 (1988)

23. Rieffel, M.A.: Induced representations of $C^{*}$-algebras. Adv. Math. 13, 176-257 (1974)

24. Stinespring, W.F.: Positive functions on $C^{*}$-algebras. Proc. Am. Math. Soc. 6, 211-216 (1955)

25. Szafraniec, F.H.: Dilations on involution semigroups. Proc. Am. Math. Soc. 66(1), 30-32 (1977)

26. Szafraniec, F.H.: Dilations of linear and nonlinear maps. In: Functions, Series, Operators, vols. I, II (Budapest, 1980), pp. 1165-1169. Colloq. Math. Soc. Janos Bolyai, 35, North-Holland, Amsterdam, (1983)

27. Sz.-Nagy, B.: Prolongement des transformations de l'espace de Hilbert qui sortent de cet espace, dans Appendice au livre "Leçons d'analyse fonctionnelle". par F. Riesz et B. Sz.-Nagy, pp. 439-573. Akadémiai Kiadó, Budapest (1955)

28. Sz.-Nagy, B., Foiaş, C.: Harmonic Analysis on Hilbert Space. North Holland, Amsterdam (1970)

29. Weron, A.: Prediction theory in Banach spaces. In: Proceedings of the Winter School of Probability, Karpacz, pp. 207-228. Springer, Berlin (1975)

30. Weron, A., Chobanyan, S.A.: Stochastic processes on pseudo-Hilbert spaces [Russian]. Bull. Acad. Polon. Ser. Math. Astr. Phys. 21, 847-854 (1973) 